ARTIGO ORIGINAL

ISSN 1677-5090

(C) 2018 Revista de Ciências Médicas e Biológicas

DOI: http://dx.doi.org/10.9771/cmbio.v17i3.26226

\title{
Perfil clínico e nutricional de pacientes hospitalizados acometidos por acidente vascular cerebral
}

\author{
Clinical and nutritional profile of stroke patients
}

\author{
Jordana Nayara Zandonai de Freitas ${ }^{1}$, Dalton Luiz Schiessel², Darla Silvério Macedo ${ }^{3}$, \\ Caryna Eurich Mazur ${ }^{*}$ \\ ${ }^{1}$ Nutricionista. Especialista em Nutrição Clínica. Faculdade Campo Real; ${ }^{2}$ Professor Doutor da Universidade Estadual \\ do Centro Oeste. Departamento de Nutrição; ${ }^{3}$ Professora Mestra do Curso de Nutrição - Faculdade Campo Real; ${ }^{4}$ \\ Professora Mestra da Universidade Estadual do Centro-Oeste. Departamento de Nutrição.
}

\begin{abstract}
Resumo
Objetivo: objetivo desse trabalho foi traçar o perfil clínico e nutricional de pacientes acometidos por acidente vascular cerebral (AVC). Metodologia: trata-se de estudo retrospectivo realizado em prontuários de pacientes internados em Hospitais de Guarapuava, Paraná no período de maio a setembro de 2016. Foram aferidos peso e estatura, e posteriormente calculado o índice de massa corporal (IMC). Ainda foi avaliada circunferência do braço (CB), aceitação da dieta e dieta prescrita pelo nutricionista. Para análise dos dados obtidos foi realizada estatística descritiva e também inferência estatística. Resultados: foram avaliados 28 pacientes que tiveram diagnóstico de AVC, com média de idade $66,2 \pm 16,3$ anos, $75 \%$ da amostra constituída por idosos e $50 \%$ do sexo feminino. A dieta oferecida com maior frequência nesses pacientes foi a dieta via sonda $(35,7 \%) ; 42,9 \%(\mathrm{~N}=12)$ foram classificados como eutróficos e 39,3\% ( $n=11$ ) como magreza. Conclusão: assim, foi possível observar que magreza foi significativa nessa amostra, mas em sua maioria prevaleceu a eutrofia, a rejeição da dieta hospitalar foi marcante principalmente em pacientes com magreza percebe-se que pacientes acometidos de AVC são vulneráveis a presença de risco nutricional.

Palavras-chave: Acidente Vascular Cerebral. Avaliação Nutricional. Estado nutricional.
\end{abstract}

\begin{abstract}
Objective: the objective of this study was to outline the nutritional clinical profile of patients with stroke. Metodology: this is a retrospective study carried out in medical records of patients hospitalized in Hospitals of Guarapuava, Paraná from May to September, 2016. Weight and height were measured, and the body mass index (BMI) was subsequently calculated. Arm circumference (AC), diet acceptance and diet prescribed by the nutritionist were also evaluated. For the analysis of the data obtained, descriptive statistics and statistical inference were also performed. Results: we evaluated 28 patients who suffered a stroke, with a mean age of 66.2 \pm 16.3 years, $75 \%$ of the sample consisting of elderly and $50 \%$ female. The diet most frequently offered in these patients was diet through nutritional therapy through a probe (35.7\%). $42.9 \%(N=12)$ were classified as eutrophic and $39.3 \%(n=11)$ as malnourished. Conclusion: thus, it was possible to observe that malnutrition was significant in this sample, but most eutrophy prevailed, the rejection of the hospital diet was marked mainly in patients with malnutrition, it is perceived that patients affected by stroke are vulnerable to the presence of nutritional risk.
\end{abstract}

Key words: Stroke. Nutrition Assessment. Nutritional Status.

\section{INTRODUÇÃO}

O acidente vascular cerebral (AVC) é uma das doenças cerebrovasculares mais frequentes, sendo a segunda causa de morte em escala mundial. Dados de 2009 mostram mais de 160 mil internações por doenças cerebrovasculares no Brasil, aonde que a taxa de mortalidade foi de 51,8 a cada grupo de 100.000 habitantes. Assim, o AVC é a principal causa de mortalidade na população adulta brasileira, $80 \%$ tem origem isquêmica e $20 \%$ hemorrágica, causando implicações medicas e sociais, como sequelas de ordem física, de comunicação, funcionais, emocionais e entre outras em mais da metade das pessoas acometidas, que precisam de

Correspondente/Corresponponding: *Caryna Eurich Mazur - Departamento de Nutrição. Universidade Estadual do Centro-Oeste. - End: Rua Simeão Varela de Sá, 3. Vila Carli. Guarapuava, Paraná, Brasil. - Tel: (42) 3629-8182. - E-mail: carynautricionista@gmail.com cuidados para sua recuperação (ALMEIDA, 2012; CARVALHO et al., 2015; CASTRO et al., 2009; FALCÃO et al., 2004).

O estudo de Carvalho et al. (2015), mostrou uma maior prevalência de AVC em idosos, foi apontado que com o passar dos anos há um aumento exponencial para esse acontecimento. Entretanto, outros autores explicam que as chances duplicam após os 50 anos de idade (CASTRO et al., 2009; PEREIRA et al., 2009). Há alguns fatores de risco além da idade que podem influenciar na etiologia do AVC como hipertensão arterial (HAS), diabetes, obesidade, fumo, etilismo entre outros. Sabe-se que com a prevenção dos fatores de risco das doenças cerebrovasculares e com a melhoria das condições socioeconômicas da população, poderia haver diminuição significativa da mortalidade por essa enfermidade (GARRITANO et al., 2012).

Desse modo, o AVC é uma síndrome neurológica de início súbito, sendo ocasionado pela interrupção do fluxo 
sanguíneo, ocasionado por trombo caracterizando um AVC isquêmico ( $\mathrm{AVCi}$ ), ou devido a extravasamento sanguíneo, caracterizando o AVC hemorrágico (AVCh) (BARROS et al., 2006; CARVALHO et al., 2015). As mais comuns manifestações clinicas do AVC são dores de cabeça intensas, acompanhada de vômitos e vertigens, alterações na fala e na linguagem, transtornos posturais e de marcha; distúrbio do sistema sensitivo e motor, ou comprometimento isolado de nervos cranianos. Já no caso de manifestações após o AVC estão presentes: hemiparesia, labilidade emocional, disfagia e consequentemente causando aspiração pulmonar, pneumonia e emagrecimento, com potencial perda de peso e desnutrição (CICHERO; MURDOCH, 2006; ITAQUY et al., 2011).

A desnutrição é comum após o episódio de AVC, havendo chances significativas de agravar-se durante o período que segue na hospitalização e também no domicílio do paciente (JAMES et al., 2005). O hipercatabolismo é elevado, como resposta ao estresse nestes doentes, e pode ser responsável pela desnutrição além da disfagia que também influencia no estado nutricional do paciente acometido por esse quadro (ITAQUY et al., 2011; PAIXÃO; SILVA; CAMERINI, et al., 2010).

Portanto, a avaliação nutricional do paciente não é tão simples, pois há implicações como impossibilidade de comunicação, dependendo do grau de acometimento do AVC, fontes escassas caso o paciente more sozinho, a avaliação do peso e altura pode ser difícil pela falta de equipamentos especiais para isso, sendo necessária avaliação antropométrica com as pregas e circunferências, a avaliação deve ser feita assim que o paciente é hospitalizado, com peso, altura e Índice de massa corporal (IMC) $\left(\mathrm{kg} / \mathrm{m}^{2}\right)$, se não for possível indica-se a medição da circunferência do braço, relacionando-a em percentis com a população específica, idade e sexo (ALMEIDA, 2007; LOSER 2005).

Visto que há um comprometimento no estado nutricional em pacientes acometidos pelo AVC, o objetivo desse trabalho foi traçar o perfil clínico e nutricional de pacientes acometidos pelo AVC dentro de duas unidades hospitalares.

\section{METODOLOGIA}

Trata-se de estudo retrospectivo efetuado com a coleta de dados em prontuários de pacientes internados em dois hospitais de Guarapuava, Paraná no período de maio a setembro de 2016. O estudo foi aprovado pelo Comitê de Ética em Pesquisa da UNICENTRO sob o número 1.593.833/2016.

Os pacientes foram pesados por meio de balança digital até $150 \mathrm{~kg}$ com precisão de $100 \mathrm{~g}$, a aferição da estatura foi por meio estadiômetro portátil com amplitude de medida de 3 metros. No caso de impossibilidade de aferir o peso e a estatura foram utilizadas estimativas de peso e estatura pelas equações de Chumlea et al. (1988) para cada gênero (SOUZA et al., 2013), através da medida desses parâmetros foi realizado o cálculo do Índice de Massa corporal (IMC). A classificação da Organização Mundial de Saúde foi proposta para os adultos da amostra (WHO, 1998), No caso dos idosos a classificação utilizada foi a preconizada por Lipschitz (1994).

Foi aferida a circunferência do braço (CB) de cada paciente no ponto médio. Os resultados obtidos foram comparados aos valores de referência proposto por Frisancho (1990).

Foi também questionado pelo nutricionista responsável a aceitação da dieta pelo paciente, visto que a mudança do estado do paciente, a hospitalização são fatores que debilitam a alimentação, a dieta hospitalar também pode ser um dos fatores marcantes para não aceitação ou aceitação pelo paciente, às vezes pela sua aparência e sabor. Foram avaliadas quais eram as dietas prescritas para cada paciente.

Para análise dos dados obtidos foi realizada estatística descritiva e também inferência estatística. Para verificar a normalidade da amostra foi aplicado o teste de Shapiro Wilk. Para comparar gêneros e estado nutricional dos pacientes foram aplicados os testes $T$ de Student e exato de Fisher, de acordo com a distribuição de normalidade. Para comparação dos três grupos de acordo com o perfil nutricional foi calculada a análise de variância (ANOVA) e Kruskal Wallis, e após verificado o valor divergente conforme post hoc de Tukey. Todos os testes foram realizados com auxílio do software SPSS ${ }^{\circledR}$ versão 22.0 para Windows.

\section{RESULTADOS E DISCUSSÃO}

Foram avaliados os prontuários de 28 pacientes com AVC, com média de idade $66,2 \pm 16,3$ anos, $75 \%(n=21)$ da amostra foi constituída por idosos e $50 \%$ ( $n=14)$ do gênero feminino.

Vários estudos demonstram que a incidência de AVC ocorre após os 60 anos de idade, assim como ocorreu no presente artigo onde a maioria dos pacientes eram idosos, sabe-se que sua indecência aumenta com a cada década de vida após os 55 anos (CARVALHO et al., 2015; CASTRO et al., 2009; PEREIRA et al. 2009).

Alguns pacientes apresentavam hábitos não saudáveis no seu cotidiano que podem influenciar na etiologia do AVC, como o tabagismo, onde aproximadamente $21 \%$ $(n=6)$ dos pacientes eram fumantes e sendo mais frequente esse hábito nos homens $(p<0,05)$ (tabela 1$)$. Muitos pacientes apresentavam doenças caracterizadas como fatores de risco para o AVC, aproximadamente $39 \%(n=11)$ eram diabéticos, e também grande parte dos pacientes apresentavam HAS sendo mais de $60 \%(n=18)$ da amostra estudada e entre as mulheres apresentava em torno de $71 \%(n=10)$ (tabela 1$)$.

Segundo dados do Instituto Nacional do Câncer (INCA) de 2007, havia 200 mil mortes anuais causadas pelo tabagismo, a prevalência do consumo de tabaco é maior nos homens do que nas mulheres, estudo de Barros et al. (2011) demonstra o mesmo resultado. Sabe-se que o tabagismo pode aumentar duas vezes o risco de AVC, sendo um fator de risco modificável (AZEVEDO, 2009). O presente estudo 
também apresentou pacientes tabagistas que foram acometidos por AVC e em sua maioria era do sexo masculino.

A HAS é um dos principais fatores etiológicos para ocorrência do AVC, sendo tanto o AVCi ou AVCh (GAGLIARDI, 2015). Na amostra estudada mais de $60 \%$ dos pacientes apresentou HAS. O diabetes também é um fator de risco para o AVC sendo uma doença frequente nas vítimas acometidas, o estudo de Silva (2011) mostrou essa realidade assim como o presente estudo também foi obtido um percentual significativo. A prevenção dessas doenças e meIhora nas condições socioeconômicas da população pode levar a uma queda significativa na mortalidade dessas enfermidades e no AVC (GARRITANO et al. 2012; PIRES, 2012).

A terapia nutricional utilizada com maior frequência nesses pacientes foi a via sonda, sendo utilizada principalmente nos homens apresentando mais de $42 \%(n=6)$; já para as mulheres predominou terapia nutricional via oral especialmente livre e pastosa $(p<0,05)$. Um fator importante que foi avaliado foi a aceitação da dieta hospitalar, apresentando um resultado negativo onde mais de 50\% $(n=16)$ considerou a dieta hospitalar ruim, entre os homens foi apresentada a pior aceitação $(p<0,05)$ (tabela 1$)$.

A nutrição é importante para a completa recuperação do paciente acometido por AVC ou qualquer outra enfermidade, sendo necessária oferta de uma alimentação adequada e individualizada para cada paciente, com aporte nutricional suficiente para haver melhora ou manutenção do estado nutricional. Como o AVC afeta as funções cerebrais, os danos ocasionados podem caracterizar déficits a nível das funções motoras, podendo ocasionar a disfagia, comprometendo assim a deglutição de alimentos pelo paciente, influenciando no seu estado nutricional (ITAQUY et al., 2011; PAIXÃO; SILVA; CAMERINI, et al. 2010; PIRES, 2012). Na pesquisa de Pires (2012) foi mostrado que os pacientes que apresentavam risco nutricional e disfagia receberam nutrição enteral, no presente estudo mais de $25 \%$ da amostra utilizou a nutrição via sonda, provavelmente pela impossibilidade de se alimentar via oral. Sabe-se que a dietoterapia mais utilizada nesses casos é a nutrição enteral (ITAQUY et al. 2011; PAIXÃO; SILVA; CAMERINI, 2010; PIRES, 2012). A dieta hospitalar normalmente é percebida como sem sabor, insossa, fria, com conotação de proibição e o próprio estado do paciente hospitalizado, fazem com que a comida oferecida seja rejeitada (BARBOSA et al., 2006a; SOUSA; GLORIA; CARDOSO, 2011) A não aceitação da dieta hospitalar foi percebida na população estudada, houve uma rejeição significativa em relação a dieta oferecida pelos hospitais, a rejeição foi maior pelos pacientes com baixo peso em relação aos pacientes eutróficos. Assim, é necessária uma atenção especial pelo nutricionista em relação a alimentação que está sendo oferecida aos pacientes para que ela seja adequada, saborosa e palatável para diminuir a rejeição (BARBOSA et al., 2006b; FERREIRA; GUIMARÃES; MARCADENTI, 2013; DIEZ-GARCIA; PADILHA; SANCHES,2012; SOUSA; GLORIA; CARDOSO, 2011). Sabe-se que há alteração do paladar na desnutrição, especialmente devido à inapetência dos pacientes em ambiente hospitalar, e ainda há alteração do paladar causada por medicamentos, o que pode contribuir para avaliação da aceitação da dieta ser considerada ruim por boa parte dos entrevistados. Uma das alternativas para minimizar essa baixa aceitação da dieta hospitalar seria a elaboração de um plano de gastronomia hospitalar e investimento especialmente financeiro da unidade hospitalar no serviço de alimentação e nutrição.

O IMC médio apresentado pelos pacientes foi de $22,3 \pm 2,8 \mathrm{~kg} / \mathrm{m}^{2}$ e os homens apresentaram maior IMC médio quando comparados às mulheres $(p<0,05)$. Em relação a circunferência do braço (CB) a média foi $26,3 \pm 3,3$ $\mathrm{cm}$, sendo que as mulheres apresentaram maior $C B$ do que os homens $(p<0,05)$ (tabela 1$)$.

Tabela 1 - Caracterização geral e de acordo com o gênero da amostra de pacientes com acidente vascular cerebral hospitalizados.

\begin{tabular}{|c|c|c|c|c|}
\hline & Total $(n=28)$ & Mulheres ( $n=14)$ & Homens $(n=14)$ & Valor de $p$ \\
\hline Idade (anos) - média/DP & $66,2 \pm 16,3$ & $67,8 \pm 16,8$ & $64,6 \pm 16,2$ & $0,11^{*}$ \\
\hline Tabagista (\%) & 21,4 & 7,1 & 35,7 & $0,01^{* *}$ \\
\hline Etilista (\%) & 7,1 & 7,1 & 7,1 & $0,93^{* *}$ \\
\hline Diabetes (\%) & 39,3 & 42,9 & 35,7 & $0,15^{* *}$ \\
\hline Hipertensão (\%) & 64,3 & 71,4 & 57,1 & $0,28^{* *}$ \\
\hline \multicolumn{5}{|l|}{ Prescrição dietoterápica } \\
\hline Livre & 25,0 & 28,6 & 21,4 & $0,07^{* *}$ \\
\hline Branda ou leve & 17,8 & 14,3 & 21,4 & \\
\hline Pastosa & 21,4 & 28,6 & 14,3 & \\
\hline Sonda nasogástrica & 35,7 & 28,6 & 42,9 & \\
\hline \multicolumn{5}{|l|}{ Aceitação da dieta hospitalar } \\
\hline Ruim & 57,1 & 42,8 & 71,4 & $0,05^{* *}$ \\
\hline Boa & 42,9 & 57,2 & 28,6 & \\
\hline $\mathrm{IMC}\left(\mathrm{Kg} / \mathrm{m}^{2}\right)$ & $22,3 \pm 2,8$ & $21,5 \pm 3,4$ & $24,5 \pm 5,1$ & $0,03 *$ \\
\hline $\mathrm{CB}(\mathrm{cm})$ & $26,3 \pm 3,3$ & $26,2 \pm 3,1$ & $22,1 \pm 2,9$ & $0,04^{*}$ \\
\hline
\end{tabular}

* Relativo ao Teste T de Student

** Relativo ao Teste exato de Fisher

$\mathrm{DP}=$ desvio-padrão 
O estado nutricional de acordo com o IMC dos pacientes foi variado, apresentando $42,9 \%$ ( $n=12$ ) classificados com eutróficos, 39,3\% ( $n=11)$ como baixo peso e um percentual baixo de $17,9 \%(n=5)$ classificados com excesso de peso/sobrepeso e obesidade (gráfico 1).

Gráfico 1 - Classificação do estado nutricional de acordo com o índice de massa corporal de pacientes com acidente vascular cerebral hospitalizados

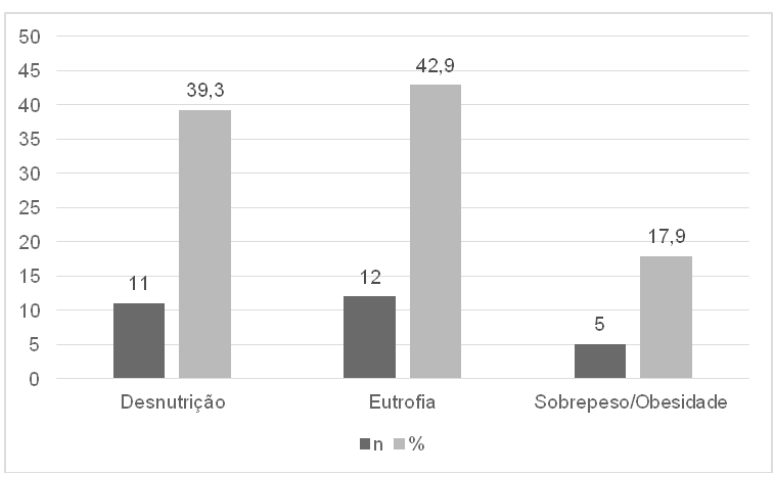

Fonte: autoria própria

Sabe-se que pacientes acometidos por AVC são caracterizados como pacientes de risco clínico, portanto o estado nutricional é um fator marcante para a reabilitação, os pacientes desnutridos/baixo peso têm maior risco sendo importante a recuperação do estado nutricional, os eutróficos precisam de acompanhamento para manutenção do seu estado nutricional, visto que são pacientes debilitados e suscetíveis a perda de peso involuntária. No estudo de Oliveira et al. (2013) os pacientes acometidos por AVC apresentaram o estado nutricional debilitado avaliado pelo IMC, apresentando um percentual significativo de pacientes desnutridos, sendo caracterizados como um grupo vulnerável e de risco, o presente estudo apresentou também um percentual significativo de pacientes desnutridos e com possível risco nutricional, apesar deste não ser avaliado com instrumento próprio.

Neste estudo os pacientes eutróficos apresentaram maior tempo de hospitalização comparados aos pacientes desnutridos e com sobrepeso/obesidade $(p<0,05)$. Em relação a aceitação da dieta, os pacientes desnutridos possuíam pior aceitação e os eutróficos apresentaram melhor aceitação $(p<0,05)$ (tabela 2$)$.

O período de internação dos pacientes pode ter influência no estado nutricional, caso não possa ser oferecido aporte nutricional adequado a eles neste período, os prejuízos poderão ser a longo prazo. A responsabilidade do nutricionista não é apenas o diagnóstico nutricional, mas é todo o tratamento nutricional, atender às necessidades nutricionais dos pacientes, procurar supri-las pela dieta e ficar atendo aos problemas nutricionais relacionados a desnutrição e ao excesso de peso (GARCIA; MERHI; PEREIRA, 2004).

Tabela 2-Comparação de perfil clínico e dietético com o perfil nutricional de pacientes com acidente vascular cerebral hospitalizados.

\begin{tabular}{|c|c|c|c|c|}
\hline & $\begin{array}{c}\text { Desnutridos } \\
\text { /baixo peso }(n=11)\end{array}$ & Eutróficos ( $n=12)$ & $\begin{array}{l}\text { Sobrepesos/Obesos } \\
\qquad(n=5)\end{array}$ & Valor de $p$ \\
\hline Idade (anos) & $71,2 \pm 16,5$ & $67,2 \pm 17,0$ & $53,0 \pm 5,1$ & $0,11^{*}$ \\
\hline Tempo de AVC (dias) & $2,4 \pm 0,2$ & $2,7 \pm 0,6 * * *$ & $1,2 \pm 0,3 * * *$ & $0,03^{*}$ \\
\hline $\mathrm{CB}(\mathrm{cm})$ & $19,2 \pm 2,4^{* * *}$ & $25,1 \pm 3,3$ & $27,1 \pm 1,4^{* * *}$ & $0,02^{*}$ \\
\hline \multicolumn{5}{|c|}{ Aceitação da dieta hospitalar (\%) } \\
\hline Ruim & 90,9 & 33,3 & 40,0 & $0,07 * *$ \\
\hline Boa & 9,1 & 66,7 & 60,0 & \\
\hline
\end{tabular}

* Relativo a Análise da Variância (ANOVA)

** Relativo a Kruskal Wallis

** Diferem estatisticamente pelo teste de Tukey com 95\% de confiança Fonte: autoria própria

Ainda, no presente estudo houve diferença na $C B$ entre os sexos, as mulheres apresentaram maior $C B$ em relação aos homens, isso pode ser justificado pois a mulher apresenta maior percentual de gordura em sua composição corporal em comparação a eles. Os homens apresentaram maior IMC em relação a elas, pois eles possuem maior percentual de massa muscular (LEITÃO et al., 2000). Neste estudo os pacientes com sobrepeso e obesidade apresentaram maior $C B$ isso é notável pois possuem maior percentual de gordura, no estudo Leitão et al. (2000) os resultados em relação a CB foram similares.

Dentre as limitações deste estudo estão a coleta em dados secundários (prontuários), muitas vezes ilegíveis e com informações limitadas. Ainda, a não estimativa de risco nutricional por instrumento próprio e também a não aferição da composição corporal para estimativas mais fidedignas de estado nutricional dos pacientes com AVC.

\section{CONCLUSÃO}

A magreza na amostra estudada é significativa, mas em sua maioria prevaleceu a eutrofia, a rejeição da dieta hospitalar é marcante principalmente em pacientes com magreza. A dieta mais oferecida é por terapia nutricional, provavelmente devido às lesões neurológicas e, ainda, a dieta hospitalar é rejeitada pela maioria dos avaliados. 
Diante disso, é possível observar que os acometidos por AVC são pacientes vulneráveis a presença de risco nutricional. Sendo assim, o acompanhamento de pacientes acometidos por AVC deve abranger todas as necessidades, e o nutricionista é importante para diagnosticar o estado nutricional e propor dietoterapia correta para minimizar problemas futuros. São necessários maiores estudos relacionados a essa temática, especialmente prospectivos ou longitudinais com maior número amostral.

\section{REFERÊNCIAS}

ALMEIDA, C. Tratamento nutricional do doente com acidente vascular cerebral na sua fase aguda. 2007. 45 f. Monografia (Especialização) Curso de Nutrição, Faculdade do Porto, Portugal, 2007.

ALMEIDA, S. Análise epidemiológica do Acidente Vascular Cerebral no Brasil. Rev. neurociênc., São Paulo, v. 20, p.481-482, 21 jan. 2012.

AZEVEDO, R. C. S. Abordagem do tabagismo: estratégia para redução de fator de risco modificável para AVC. Comciência, Campinas, v. 109, 2009.

BARBOSA, M. F. P. et al. Correlação entre os achados clínicos da deglutição e os achados da tomografia computadorizada de crânio em pacientes com acidente vascular cerebral isquêmico na fase aguda da doença. Arq. neuropsiquiatr., São Paulo, p. 1009-1010, 8 jun. 2006b.

BARBOSA, M. F. P. et al. Do cuidado nutricional ao cuidado alimentar: percepção de pacientes sobre a refeição hospitalar. Nutrição em Pauta, Rio de Janeiro, v. 79, p. 48-54, 2006a.

BARROS, A. J. D. et al. Tabagismo no Brasil: desigualdades regionais e prevalência segundo características ocupacionais. Ciênc. Saúde Colet., Rio de Janeiro, v. 9, n. 16, p. 3707-3716, 2011.

CARVALHO, M. A. et al. Epidemiologia dos acidentes vasculares encefálicos atendidos por meio do serviço de atendimento móvel de urgência. Rev. Enferm. UFPR on line., Recife, v. 3, n. 9, p. 1015-1021, 2015. Disponível em:<file:///D:/atendimento/Downloads/7189-68765-1-PB. pdf>. Acesso em: 23 fev. 2017.

CASTRO, J. A. B. de et al. Estudo dos principais fatores de risco para acidente vascular encefálico. Rev. Soc. Bras. Clín. Méd., São Paulo, v.7, n. 3, p. 171-173, 2009.

CHUMLEA, W. M. C. et al. Prediction of body weight for the nonambulatory elderly from anthropometry. J. Am. Diet Assoc., Chicago, v. 88, n.5, p. 564-568, 1988.

CICHERO, J. A.; MURDOCH, B, E. Dysphagia: foundation, theory and practice London: John Wiley \& Sons, 2006.

FALCÃO, I. V. et al. Acidente vascular cerebral precoce: implicações para adultos em idade produtiva atendidos pelo Sistema Único de Saúde. Rev. Bras. Saúde Mat. Infant., Recife, v. 4, n. 1, p. 95-101, 2004.

FERREIRA, D.; GUIMARÃES, T. G.; MARCADENTI, A. Aceitação de dietas hospitalares e estado nutricional entre pacientes com câncer. Revista Einstein, são Paulo, v. 11, n 1, p. 41-46, 2013.

GAGLIARDI, R J. Prevenção primária da doença cerebrovascular. Diagn Tratamento, São Paulo, v. 20, n.3, p. 88-94, 2015.

GARCIA, R. W. D.; MERHI, V. A. L.; PEREIRA, A. M. Estado nutricional e sua evolução em pacientes internados em clínica médica. Rev. bras. nutr. clín., Porto Alegre, v. 19, n. 2, p. 59-63, 2004.

DIEZ-GARCIA, R. W.; PADILHA, M.; SANCHES, M. Alimentação hospitalar: proposições para a qualificação do serviço de alimentação e nutrição, avaliadas pela comunidade científica. Ciênc. Saúde Colet., Rio de Janeiro, v. 17, n. 2, p. 473-480, 2012.

FRISANCHO, A. R. Anthropometric standards for the assessment of growth and nutritional status. Ann Arbor: The University of Michigan Press, 1990.

GARRITANO, C. R. et al. Análise da Tendência da Mortalidade por Acidente Vascular Cerebralno Brasil no Século XXI. Arq. bras. cardiol, São Paulo, 2012.

INSTITUTO NACIONAL DE CÂNCER (INCA). Tabagismo, dados e números, 2007. Disponível em: $<$ http://www.inca.gov.br/releases/press_release_view_arq.asp?ID=1493> Acesso em: 23 fev. 2017.

ITAQUY, R. B. et al. Disfagia e acidente vascular cerebral: relação entre o grau de severidade e o nível de comprometimento neurológico. J. Soc. Bras. Fonoaudiol., São Paulo, v. 23, n. 4, p. 385-9, 2011.

JAMES, R. et al. Nutrition support (tube feeding) as a rehabilitation intervention. Arch. Phys. Med. Rehabil., Philadelphia, v. 85, n. 12, 2005.

LEITÃO, M. B. et al. Posicionamento oficial da Sociedade Brasileira de Medicina do Esporte: atividade física e saúde na mulher. Rev. Bras. Med. Esporte, Niteroi, v. 6, n.6, Nov./Dec. 2000.

LIPSCHITZ, D.A. Screening for nutritional status in the elderly. Prim. care, Oxford, v. 21, n.1, p.55-67, 1994.

LOSER, C. et al. ESPEN guidelines on artificial enteral nutrition - Percutaneous endoscopic gastrostomy (PEG). Clin Nutr., New York, v. 24, n. 5, p. 848-861, 2005.

OLIVEIRA, A. R. S. et al. Avaliação de pacientes com acidente vascular cerebral acompanhados por programas de assistência domiciliária. Rev. Esc. Enferm. USP, São Paulo, v. 47 n.5, 2013.

PAIXÃO, C. T.; SILVA, L. D.; CAMERINI, F. G. Perfil da disfagia após um acidente vascular cerebral: uma revisão integrativa. Rev. RENE, Fortaleza, v. 11, n. 1, p. 181-190, 2010.

PEREIRA, A. B. C. N. da G. et al. Prevalência de acidente vascular cerebral em idosos no Município de Vassouras, Rio de Janeiro, Brasil, através do rastreamento de dados do Programa Saúde da Família. Cad. Saúde Pública, São Paulo, v. 25, n.9, p.1929-1936, 2009.

PIRES, C V. Avaliação nutricional na admissão do doente com AVC. 2012. Tese (Mestrado em enfermagem de reabilitação) - Escola Superior de Saúde de Bragança, Bragança, 2012.

SILVA, M. A. C. Níveis de glicémia capilar e prognóstico do doente com AVC. 2011. Dissertação (Mestrado em Medicina) - Universidade da Beira Interior, Portugal, 2011.

SOUSA, A, A, de.; GLORIA, M. de S.; CARDOSO, T. S. Aceitação de dietas em ambiente hospitalar. Rev. Nutr., v. 24, n. 2, p. 287-294, Campinas, 2011.

SOUZA, R. et al. Avaliação antropométrica em idosos: estimativas de peso e altura e concordância entre classificações de IMC. Rev. bras. geriatr. gerontol., Rio de Janeiro, v. 16, n. 1, p. 81-90, 2013.

WORLD HEALTH ORGANIZATION. Obesity: preventing and managing the global epidemic. Geneva: WHO, 1998. (Technical Report Series, n. 894).

Submetido em: 10/04/2018

Aceito em: 19/10/2018 